\title{
Estrategias de marketing el conocimiento de los trámites vía online del Servicio de Rentas Internas
}

\section{Marketing strategies knowledge of online procedures of the Internal Revenue Service}

\author{
MSc, Achi González Yolanda Elizabeth ${ }^{1}$ \\ yoly achi@hotmail.com \\ PhD, Maridueña Arroyave, Milton Rafael ${ }^{2}$ \\ mmariduena@itsve.edu.ec
}

Recibido: 1/12/2018; Aceptado: 1/02/2019

\section{RESUMEN}

El principal objetivo del presente artículo científico es poner al conocimiento de la población en general los servicios o trámites que pueden ser realizados directamente por vía internet en el portal web del Servicio de Rentas Internas, sin necesidad de que los usuarios acudan a las agencias y sucursales del SRI en las distintas partes del Ecuador. Se realizan estrategias de marketing que serán aplicadas en el objeto de estudio. Para la investigación de campo a realizar, se tomó en cuenta como población a todas aquellas personas naturales no obligadas a llevar contabilidad de la parroquia Tarqui de la ciudad de Guayaquil. El número total fue obtenido de los registros propios del sistema del Servicio de Rentas Internas. La misma investigación de campo dio como resultados que las personas naturales y jurídicas no tenían conocimiento de que existían algunos trámites que pueden realizar a través del internet en el portal web del SRI. Esto se da debido a la poca efectividad que ha tenido la promoción de estos servicios por parte del Servicio de Rentas Internas. Es por ello que existe la necesidad de que se mejoren dichas estrategias de promoción para así ir dando solución a estos inconvenientes.

Palabras Clave: trámites online, Servicios de Rentas Internas, usuarios, estrategias, promoción

\section{ABSTRACT}

The main objective of this scientific article is to inform the population in general about the services or procedures that can be performed directly through the

\footnotetext{
${ }^{1}$ Universidad de Guayaquil. Ecuador

2 Universidad de Guayaquil. Ecuador
} 


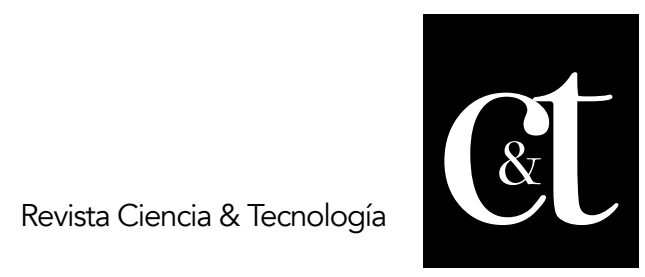

No. 22, 30 de abril de 2019

ISSN impreso: 1390 - 6321

ISSN online: 2661 - 6734

Internet in the Internal Revenue Service web portal without the need for users to go to the agencies and branches of the SRI in the different parts of Ecuador, marketing strategies are carried out that will be applied in the object of study, for the field investigation to be carried out, it was taken into account as a population all those natural and legal persons of the city of Guayaquil have RUC, the total number was obtained from the records of the Internal Revenue Service system, the same field investigation gave as results that natural and legal persons had no knowledge that there were some procedures that can be done through the internet in the SRI web portal, this is due to the lack of effectiveness that the prom These services are offered by the Internal Revenue Service, which is why there is a need to improve these promotion strategies in order to solve these problems.

Keywords: online procedures, Internal Revenue Services, users, strategies, promotion

\section{Introducción}

El avance de la nueva tecnología ha tenido lugar desde el comienzo de la historia humana. Desde la invención de elementos como la lanza y los cuchillos hechos de rocas y palos para ayudar en la captura y muerte de animales para alimento, hasta elementos como la primera imprenta y la computadora. El avance en la tecnología ha sido excepcionalmente rápido en los siglos XX y XXI. Dado que la tecnología electrónica y las máquinas se producen y mejoran constantemente, es muy probable que, junto con los aspectos positivos de estos nuevos avances, las personas también consideren los aspectos negativos y busquen criticar las nuevas tecnologías.

Cada día, otra compañía saca algo más avanzado en un intento de ganar la guerra del consumidor contra otra compañía. Este consumismo está impulsando la tasa de avance cada vez más rápido. El desarrollo del iPhone por Apple es un ejemplo de esto. Apple sacó un teléfono que literalmente podía hacer todo y ninguna otra compañía en el mercado de la telefonía móvil podía compararlo. No solo podía hacer llamadas telefónicas y mensajes de texto, sino que también podía ver videos, tomar fotos y videos, escuchar música, conectarse a Internet, tenía aplicaciones para casi cualquier cosa.

La tecnología es impulsada por la demanda del consumidor. Los teléfonos, las computadoras y los relojes hacen más que solo su propósito innato ahora, y mientras el público entregue el efectivo, la tecnología continuará expandiéndose, razón por la cual avanza a un ritmo excepcional. En el pasado solía tomar siglos para que la innovación echara raíces y hoy los avances ocurren casi a diario.

Los efectos del cambio tecnológico en la estructura económica global están creando enormes transformaciones en la forma en que las empresas y las naciones organizan la producción, comercian bienes, invierten capital y desarrollan nuevos productos y procesos. Las tecnologías de información sofisticadas permiten la comunicación instantánea entre las operaciones lejanas de las empresas globales.

Los nuevos materiales están revolucionando sectores tan diversos como la construcción y las comunicaciones. Las tecnologías de fabricación avanzadas han 


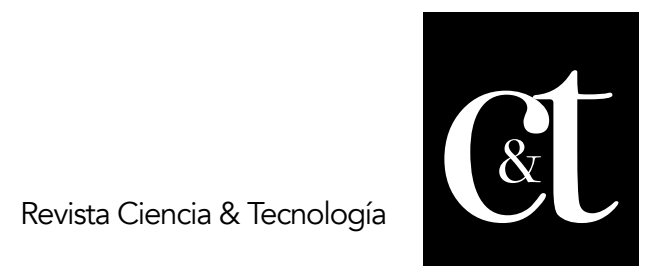

No. 22, 30 de abril de 2019

ISSN impreso: 1390 - 6321

ISSN online: 2661 - 6734

alterado los patrones de productividad y empleo de larga data. La mejora del transporte aéreo y marítimo ha acelerado en gran medida el flujo mundial de personas y bienes. Todo esto ha creado y ordenado una mayor interdependencia entre empresas y naciones. El rápido ritmo de innovación y la dinámica de los flujos de tecnología significan que la ventaja comparativa es de corta duración.

Para maximizar los retornos, se buscan acuerdos tales como fusiones transnacionales y acuerdos de producción compartida para unir a los socios con intereses y fortalezas complementarias. Esto permite que los países desarrollados y en desarrollo aprovechen la tecnología de manera más eficiente, con la expectativa de crear niveles de vida más altos para todos los involucrados.

El desarrollo de Internet ha alterado significativamente las operaciones diarias de una empresa; incluyendo cómo se comunican entre ellos y su audiencia. La información se puede transmitir fácilmente a cualquier destino en cuestión de segundos. Internet se ha convertido en una herramienta esencial para el marketing y la publicidad. Una empresa puede presentarse a los clientes con el uso de un sitio web o anuncios en línea. Muchas empresas ahora usan Internet como medio para informar a los clientes sobre sus promociones actuales. Esto puede ser muy beneficioso para las empresas que apuntan a un público más joven.

\section{Desarrollo}

La comunicación y la interacción con los clientes son vitales para cualquier negocio. Internet se ha asegurado de que esto pueda lograrse fácilmente. Las empresas pueden comunicarse e interactuar con los clientes por correo electrónico o mensajería instantánea. La telefonía por Internet es ahora un método popular de comunicación y es utilizado frecuentemente por las empresas para llevar a cabo reuniones virtuales con clientes y otras empresas. El uso de Internet también facilita que las empresas envíen mensajes a las personas que trabajan dentro de la organización.

Internet ha simplificado la forma en que las empresas recopilan y registran la información. Pueden realizar investigaciones efectivas buscando en la web o utilizando bases de datos en línea. Luego se puede hacer un registro electrónico de la información recopilada. También se puede obtener información importante, como el estado de la bolsa de valores. Además, las empresas que tienen presencia internacional pueden usar Internet para realizar operaciones y comunicarse con personas en oficinas ubicadas en diferentes lugares del mundo. Las transacciones y los pagos ahora también pueden realizarse en línea, lo que simplificó y aceleró el proceso de pago.

La World Wide Web funciona las 24 horas del día, los siete días de la semana. Las empresas que venden productos prefabricados se benefician más de esta ventaja. Al crear una tienda de Internet, estos emprendedores tienen la capacidad de mantener una tienda virtual de venta minorista que nunca se cierra, ofreciendo al propietario la posibilidad de ganar dinero literalmente mientras duerme. Internet ha creado una comunidad global de pares.

Durante al menos los últimos veinte años, el papel desempeñado por los gobiernos se ha alejado cada vez más de la prestación directa de servicios hacia la regulación y la gobernanza de los servicios prestados por una multiplicidad de entidades privadas y sin fines de lucro. Los gobiernos han incorporado la reforma y la innovación 


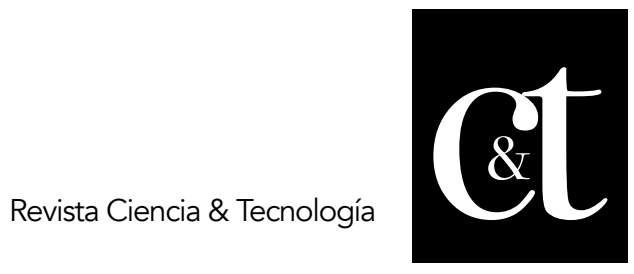

No. 22, 30 de abril de 2019

ISSN impreso: 1390 - 6321

ISSN online: 2661 - 6734

del sector público en sus programas, esforzándose por ofrecer servicios públicos más eficientes y efectivos, a fin de satisfacer las crecientes expectativas de los ciudadanos con presupuestos públicos cada vez más reducidos.

En el caso de las empresas públicas el uso del internet se ha convertido en una herramienta exitosa de comunicación e interacción con los usuarios, en instituciones como el IESS o hasta en instituciones educativas de gobierno el uso del internet o también de las conocidas tecnologías de información y comunicación (TICs) han sido aplicadas de forma eficiente, como por ejemplo permitiendo al usuario obtener una clave para su afiliación al IESS como también para la obtención de una matrícula para un menor de edad.

Las tecnologías de la información y la comunicación han sido reconocidas desde hace tiempo como una herramienta estratégica clave para permitir estas reformas. Desde finales de los años 90, ha sido fundamental para impulsar las políticas de reforma del gobierno. Se han realizado importantes inversiones humanas y financieras en el Ecuador con el objetivo de ofrecer servicios públicos de mejor calidad, reducir los tiempos de espera y mejorar la rentabilidad, aumentar la productividad y mejorar la transparencia y la rendición de cuentas.

El SRI es una de las instituciones del Estado cuya aplicación de las tecnologías de información y comunicación se han convertido en las que más rápida adaptación ha tenido en su portal web. Esto debido a que actualmente en aquel portal, pueden realizarle diferentes tipos de trámites, el cual le permite al usuario no pasar por las largas colas que anteriormente sucedía, para realizar un trámite en específico, sin embargo mucha gente desconoce de qué tipo de servicios o tramites son los que se pueden realizar a través de la internet. Gran parte de la evasión fiscal que existe, se debe a una gran falta de conocimiento, como lo mencionan Arévalo y Crespo (2016).

El desconocimiento, puede causarse por la poca promoción de estas formas de tramitología en los diversos informativos del país. Existen personas que de hecho aún se acercan hacia las instalaciones del SRI en la ciudad de Guayaquil a realizar diligencias que ya no son hechos de modo presencial. Es ahí en donde recién se enteran que pueden realizar algunos trámites en específico dentro del portal web del SRI. Ante esta situación es necesario aplicar estrategias de marketing para dar a conocer los trámites que pueden ser realizados vía internet.

Para todas las empresas, ya sea una modesta empresa de nueva creación o un gigante multinacional, una estrategia de marketing efectiva puede servir como un mapa de ruta crucial para todo el negocio. Al desarrollar y establecer una estrategia de mercadotecnia coherente y bien considerada, las organizaciones pueden promover sus negocios, cortejar a los tipos de clientes correctos y asignar sus recursos correctamente, a la vez que salvaguardan la reputación de la empresa.

Como tal, las estrategias de marketing efectivas están compuestas por numerosas facetas que las empresas deben considerar en todo momento. El valor para el accionista, el riesgo para la reputación y el impacto de la tecnología digital son tres de las consideraciones más importantes que las empresas deben tener en cuenta al intentar comercializar sus servicios a la audiencia adecuada. Sin lugar a dudas, una estrategia de marketing integral y efectiva es algo que las empresas deben emplear independientemente de su tamaño. 
Las expectativas de los consumidores deben tomarse en consideración, y un análisis detallado de las operaciones de una empresa permitirá a las empresas ver sus negocios desde la perspectiva del cliente o del cliente. La creación de una estrategia de marketing efectiva es a menudo la tarea del equipo directivo superior de una empresa (Ferrell y Hartline, 2014). Mediante la entrega de una estrategia de marketing integral, las empresas ayudarán a crear un enfoque de marketing más centrado y de mayor alcance comercial.

Sin embargo, es crucial que la estrategia sea multifacética, realista e implementada de manera consistente a lo largo del tiempo. Una estrategia de mercadotecnia efectiva también debe servir para cerrar cualquier brecha que pueda existir entre la sala de juntas y los comercializadores responsables de diseminar el mensaje de la compañía (Armijos, 2019). Las estrategias de mercadotecnia a menudo requieren disciplina y gobernabilidad a nivel de toda la compañía para garantizar que se implementen de manera efectiva y eficiente.

Principales estrategias para dar a conocer un servicio Impresión y artes gráficas Dependiendo del tipo de mensaje que desea comunicar a los usuarios del SRI, los medios impresos ofrecen diferentes opciones, que incluyen: folletos, tarjetas de presentación, anuncios en periódicos y revistas.

Los folletos, carteles y empaques son una forma rentable de proporcionar una variedad de mensajes e información detallada sobre sus productos y servicios.

Las tarjetas de negocios pueden usarse para respaldar sus actividades de red y brindar a los clientes potenciales la información que necesitan para contactarlo.

La publicidad en los periódicos locales es una forma de llegar a las personas de su comunidad y las expone reiteradamente a su mensaje para crear una presencia local más sólida para su empresa.

Las revistas tienen la ventaja de dirigirse a un público más específico de suscriptores que están interesados en los temas que cubre.

Medios Electrónicos

Medios electrónicos es un término general para cualquier medio que requiera un dispositivo electrónico para acceder al contenido. Algunas de las formas más comunes de medios electrónicos incluyen televisión, radio, internet y contenido para dispositivos móviles.

El contenido de la televisión capta más tiempo de audiencia que cualquier otro medio y está dirigido a las audiencias hogareñas.

La radio es rentable, y la audiencia generalmente es fiel al formato del programa de la estación.

Internet ofrece una variedad de formas diferentes de comercializar un o 
servicio en un sitio web o por correo electrónico.

$>$ Los teléfonos celulares y teléfonos inteligentes permiten tácticas de marketing que permiten llegar a los clientes directamente desde sus móviles.

$>$ El marketing de redes sociales fomenta la interacción en línea entre sus clientes y su empresa mediante varios sitios de redes sociales.

Ideas promocionales adicionales

Los obsequios promocionales, como bolígrafos, llaveros y calendarios, se pueden entregar a personas y grupos que asisten personalmente a las instalaciones del SRI, sin saber los tramites que pueden realizarse vía internet, a ellos se les puede entregar los obsequios para ayudar a crear una opinión positiva de SRI y mejorar la reputación del mismo.

Las redes y la participación de la comunidad también pueden respaldar la promoción de un servicio, como el caso de los tramites online del SRI. Puede hacer nuevos contactos y llegar a clientes potenciales al participar en ferias comerciales, conferencias, actividades comunitarias y otros eventos de redes. Hablarle a la gente sobre el servicio puede ser una de las maneras más efectivas de promocionar el mismo.

La planificación de la estrategia de marketing y publicidad ayudará al SRI a determinar la mejor manera de promocionar su servicio, le permitirá medir el éxito en relación con los objetivos establecidos y brindarle una idea más clara de dónde puede necesitar ajustes la estrategia ejecutada.

\section{Materiales y Métodos}

Para cumplir con el objetivo del presente artículo científico ser ha realizado una investigación exploratoria dirigida a las personas naturales no obligadas a llevar contabilidad de la parroquia Tarqui de la ciudad de Guayaquil. Según el servicio de rentas internas (S.R.I.) a través de su representante (Nathalie Heredia Gallegos) afirma que en la parroquia Tarqui del cantón Guayaquil las personas naturales que declaran el impuesto al valor agregado (I.V.A.) sea su presentación mensual o semestral en el 2017 fue de 73,278 contribuyentes. Por este concepto, en base a esta condición y resultados obtenidos, se tomará como dato la población.

Al aplicar la fórmula para la obtención de la muestra, se determina que se harán se realizarán encuestas a un total de 118 personas naturales que no obligadas a llevar contabilidad de la parroquia Tarqui de la ciudad de Guayaquil. La investigación de campo dará como resultado, información relevante, relacionada con el conocimiento que tiene las personas sobre los tramites que pueden realizarse de forma online en el portal web del SRI (SRI, 2016), para el cumplimiento de los objetivos planteados para el presente artículo científico.

\section{Resultados}

De los datos obtenidos de la encuesta se identifica que la gran mayoría de las personas naturales no obligadas a llevar contabilidad, no reciben información por parte del Sistema de Rentas Internas, acerca de alguna modificación en las actividades económicas a la que se dedican, o información sobre actualizaciones que se necesitan 


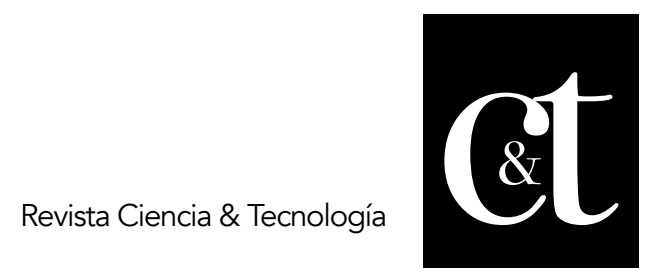

No. 22, 30 de abril de 2019

ISSN impreso: 1390 - 6321

ISSN online: 2661 - 6734

registrar, ni mucho menos de información acerca de trámites que deban realizar.

$$
\begin{aligned}
& n=\frac{73,278(0.5)^{2}(1.96)^{2}}{(73,278-1)(0.09)^{2}+(0.5)^{2}(0.5)(1.96)^{2}} \\
& n=\frac{70,376.1912}{593.5437+0.4802} \\
& n=\frac{70,376.1912}{594.0239} \\
& n=118 \text { Personas }
\end{aligned}
$$

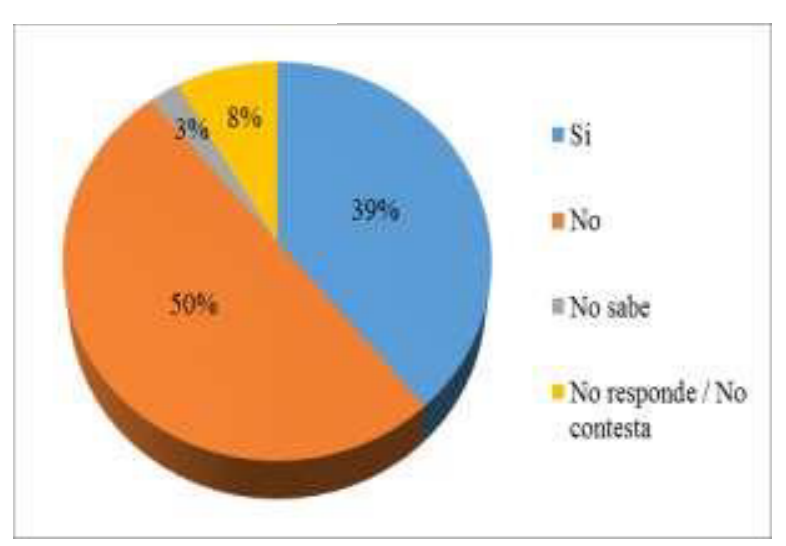

\section{Gráfico 1. Recibimiento de información por parte del SRI}

Fuente: Elaboración propia

Puede ser concluido que según los estudios realizados en el Tarqui Cantón la parroquia de Guayaquil, la diferencia entre personas naturales no obligadas a llevar contabilidad, en una gran parte quien asistió a las capacitaciones (los entrenamientos) y los que no fueron a la educación (al entrenamiento) es mínimo. En cambio los contribuyentes dijeron que ellos van a una tercera persona a hacer su declaración. Es más, esta opción permite determinar el volumen de contribuyentes por un tercero. La asignación por un profesional puede influir en el manejo y la manipulación de información de datos en la declaración y la presentación de distintos trámites.

Puede concluirse que según los estudios realizados en la parroquia Tarqui de la ciudad de Guayaquil, la mayoría de personas naturales no obligadas a llevar contabilidad indicaron que si conocían que existían tramites que se los podían realizar en el portal web del SRI (SRI, 2016). Sólo un cuarto de los encuestados respondieron positivamente a esta pregunta, mientras que en su gran mayoría las personas no obligadas a llevar contabilidad indicaron que no conocían que se podía realizar algún tipo de tramite vía online en el portal del Servicio de Rentas Internas. 


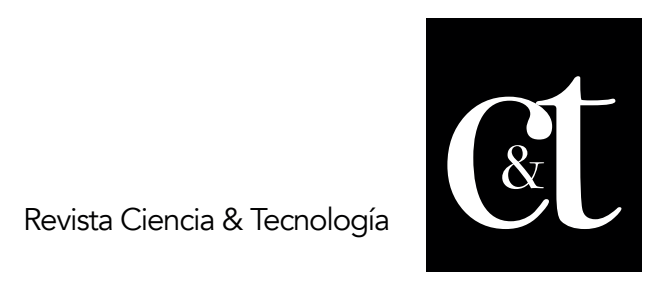

No. 22, 30 de abril de 2019

ISSN impreso: 1390 - 6321

ISSN online: 2661 - 6734

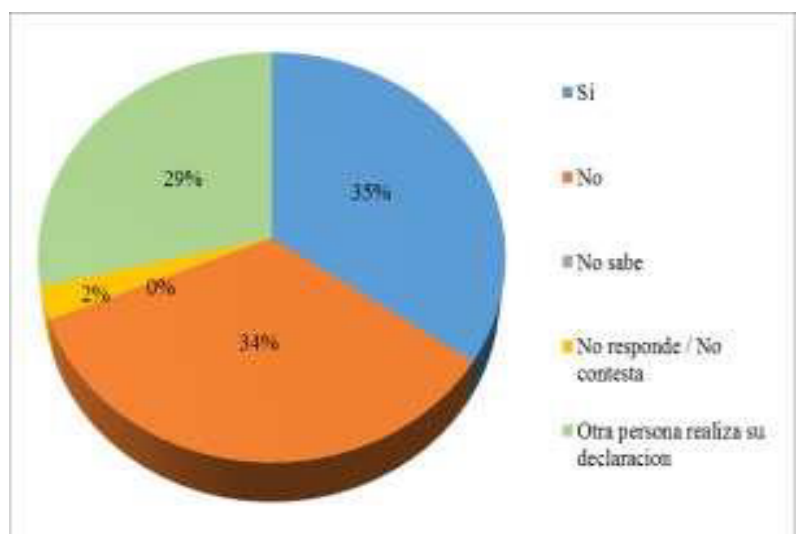

Gráfico 2. Asistencia a capacitaciones por parte del SRI Fuente: Elaboración propia

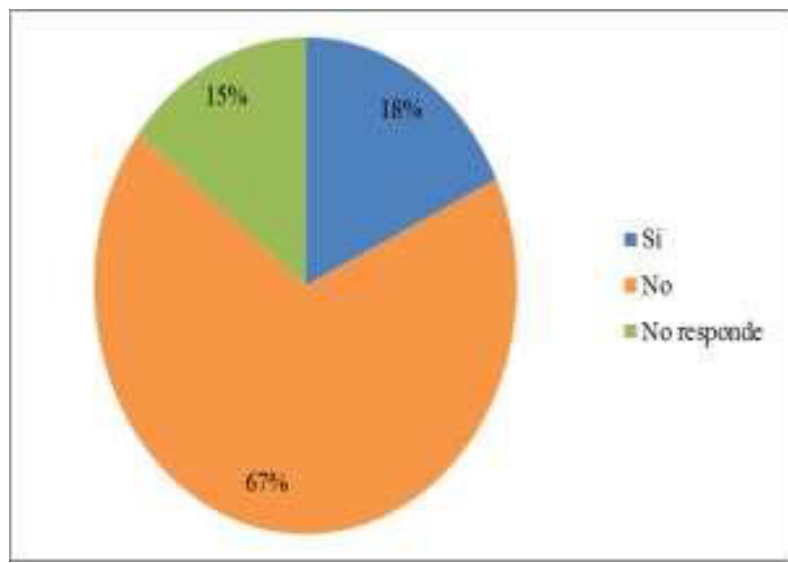

Gráfico. 3 Conocimiento sobre realización de trámites por medio del portal web del SRI

Fuente: Elaboración propia

La gran mayoría de las personas naturales no obligadas a llevar contabilidad indicaron que no han realizado algún trámite online que tenga que ver con el Servicio de Rentas internas, como ha sido la tendencia. Muchos se han notado sorprendidos de conocer que vía online se pueden realizar varios trámites más allá de la declaración de impuesto. Es necesario que se mejore esta situación y que los usuarios conozcan más a fondo las diligencias que pueden realizarse en el portal web del SRI, puesto que solo una mínima parte de los encuestados manifestaron que si han realizado tramites. 

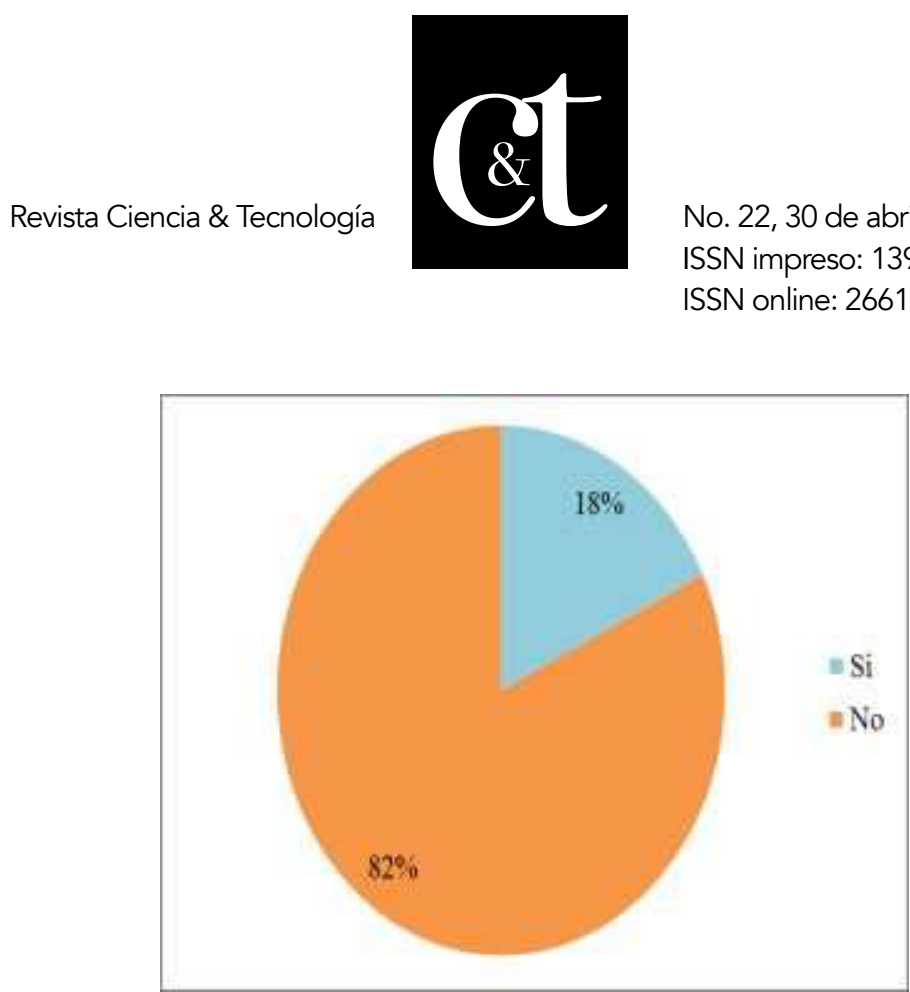

\section{Gráfico 4. Ha realizado un trámite por medio del portal web del SRI Fuente: Elaboración propia}

De los datos obtenidos de la encuesta, se identifica que la gran mayoría de las personas naturales no obligadas a llevar contabilidad de la parroquia Tarqui de la ciudad de Guayaquil, solo reconocían que las declaraciones del impuesto al valor agregado (IVA) y la declaración del Impuesto a la renta (IR) se podían realizar vía internet. Fuera de estas dos opciones el resto no conocía algún otro trámite que se podía realizar en el portal web del Servicio de Rentas Internas.

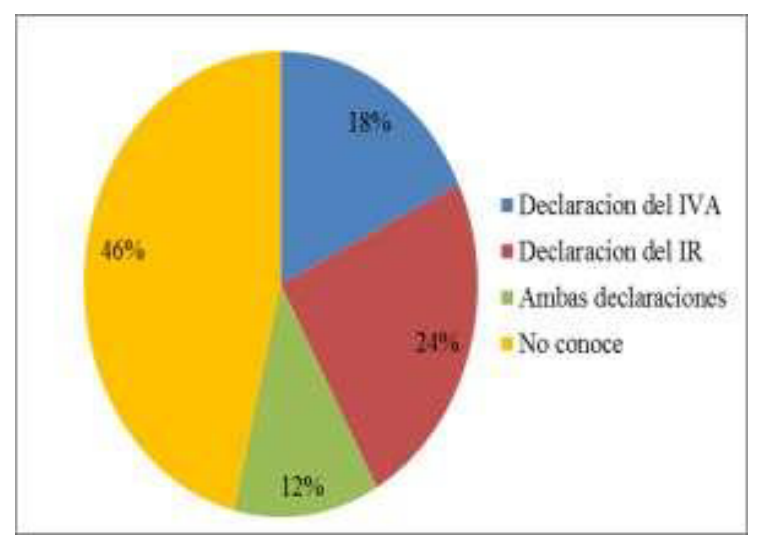

\section{Gráfico 5. Tipos de trámites que conocía que se realizan a través del portal web del SRI \\ Fuente: Elaboración propia}

De los datos de la encuesta dirigida a las personas naturales no obligadas a llevar contabilidad de la parroquia Tarqui de la ciudad de Guayaquil, la gran mayoría de los mismos manifestó no haber visto anteriormente a la realización de esta investigación de campo una publicidad o alguna promoción acerca de los tramites que podían realizarse a través del portal web del SRI. Solo una mínima parte de los encuestados respondieron positivamente ante esta pregunta. Siendo así, se justifica el hecho de que muchos usuarios asistan directamente a las instalaciones del SRI a realizar diferentes tipos de trámites. 


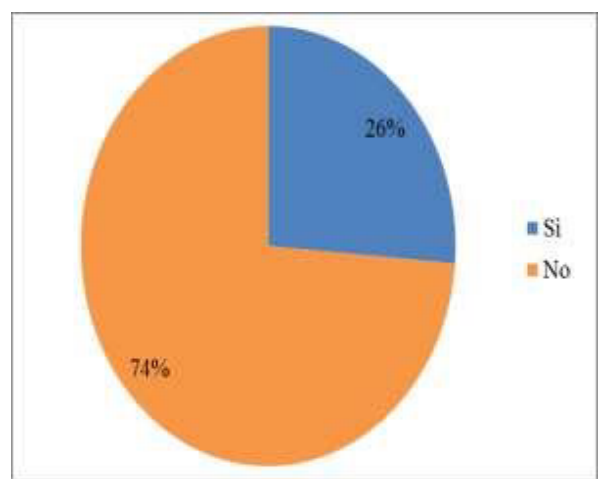

\title{
Gráfico 6. Visualización de publicidad de trámites se realizan a través del portal web del SRI \\ Fuente: Elaboración propia
}

\begin{abstract}
Discusión
El marketing estratégico es visto por varios autores incluyendo a Kotler y Levy (1967) como un proceso que consiste en: analizar, competir en el mercado y analizar los factores comerciales que afectan a las corporaciones y sus unidades de negocio, identificar oportunidades de mercado y amenazas, además de complementarlos con pronosticar tendencias futuras en áreas comerciales de interés para la empresa, y participar en establecer objetivos y formular estrategias de unidades corporativas y de negocios.
\end{abstract}

El marketing en línea es difícil. Implica entrelazar un conjunto de conocimiento complejo y altamente técnico en una amplia gama de habilidades. No solo se hace referencia de la optimización de motores de búsqueda, pues cada empresa que ofrece un servicio vía internet es más veloz y eficiente en una plataforma de internet específica que en todas. También se sabe lo importante que es promocionar algún servicio en línea para ganar visibilidad y llegar al público objetivo.

Hay varias formas en las que puede comercializar un servicio. El marketing en línea ofrece a las empresas un desafío emocionante y una forma de llegar a personas de todo el mundo en una instancia. En realidad, su postura de marketing reflejará el tamaño de su empresa, el presupuesto con el que está trabajando y el tipo de personas que está tratando de atraer como clientes.

En general, los productos o los servicios de una empresa no son vendidos solos, siempre tienen que ser promocionados de algún modo, hacerlos atractivos a clientes, sólo así conocen un producto o servicio o fomentan la acción de uso de tal servicio. La forma en la cual una empresa se comunica, es importante en la atracción de clientes, la herramienta más común es la realización de promoción del producto 0 servicio para la llegada del producto al mercado objetivo (Gutiérrez y Nava, 2016).

Es necesario establecer las estrategias de marketing para los objetos de estudios analizados, los cual permitirá que se registre el aumento requerido en los ingresos de las personas naturales no obligadas a llevar contabilidad de la parroquia Tarqui de la ciudad de Guayaquil. Son de estas acciones estratégicas que dependerá que el mercado meta haga uso de los servicios que presta el Servicio de Rentas Internas a 
través del internet en su portal web.

Sin embargo, es importante tener en cuenta que intentar cualquiera de estos métodos de marketing de bajo presupuesto en línea no garantiza su éxito. De hecho, la mayoría de ellos requieren algún conocimiento serio y profundamente arraigado en sus respectivas áreas. Cualquier estrategia debe fortalecer la labor del SRI (Estrella, 2014).

\section{Conclusiones}

El SRI es una de las instituciones que más adaptada está a la tecnología de información y comunicación, puesto que actualmente en aquel portal, pueden realizarle diferentes tipos de trámites, el cual le permite al usuario no pasar por las largas colas que anteriormente sucedía, para realizar un trámite en específico. Sin embargo, mucha gente desconoce de qué tipo de servicios o trámites son los que se pueden realizar a través del internet.

La poca promoción y difusión de los trámites que pueden realizarse vía online a través del portal web del SRI, es el principal causante del desconocimiento de los servicios en línea que ofrece el SRI. Existen personas que de hecho aún se acercan hacia las instalaciones del SRI en la ciudad de Guayaquil a realizar diligencias que ya no son hechos de modo presencial.

Las redes y la participación de la comunidad también pueden respaldar la promoción de un servicio, como el caso de los tramites online del SRI. Puede hacer nuevos contactos y llegar a clientes potenciales al participar en ferias comerciales, conferencias, actividades comunitarias y otros eventos de redes. Hablarle a la gente sobre el servicio puede ser una de las maneras más efectivas de promocionar el mismo.

Claramente, la forma más importante en que se puede promocionar los servicios que ofrece el SRI para realizar trámites online desde su portal web es creando un blog donde pueda publicar y compartir contenido de alta calidad que agregue una gran cantidad de valor de forma periódica. Esta es definitivamente una estrategia a muy largo plazo, y no valdrá la pena de la noche a la mañana, pero cada emprendedor necesita comprender la importancia de adoptar este método de comercialización en línea.

\section{Recomendaciones}

Si bien puede resultar contraproducente crear un tutorial en video que enseñe algo, es con mucho, una de las mejores maneras de no solo establecerse como una promoción puesto que comercializar un servicio en línea, lleva tiempo crear una audiencia y seguirla. Pero, los videos tutoriales ofrecen uno de los mejores métodos para atraer a la demografía correcta que estaría interesada en lo que sea que esté ofreciendo.

El marketing por correo electrónico no es solo un método de bajo costo para difundir la palabra proverbial en ningún negocio, sino que también ofrece uno de los mejores rendimientos de la inversión de su tiempo. Sin embargo, para tener éxito con el marketing por correo electrónico, debe atraer a los clientes adecuados a su sitio web, algo que no es tan simple como parece. 


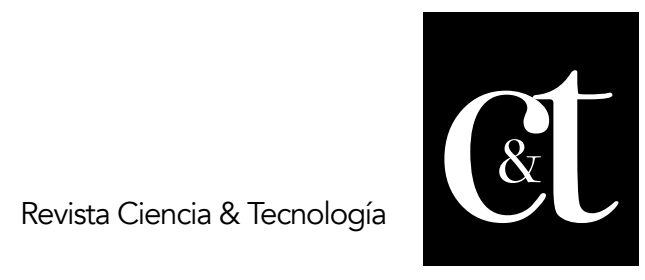

No. 22, 30 de abril de 2019

ISSN impreso: 1390 - 6321

ISSN online: 2661 - 6734

Al crear contenido atractivo como infografías y otros tutoriales en línea, puede atraer a los clientes correctos. Sin embargo aún se tiene trabajo por delante, puesto que será necesario que la página web vaya teniendo que compartir información útil con ellos de forma periódica al intentar realizar la venta.

Hoy en día, con el poder siempre presente de las redes sociales, se puede llegar instantáneamente a miles de personas de todo el mundo en cualquier momento. Pero también es recomendable tomar en cuenta que los algoritmos y la visibilidad están trabajando en contra si es que no se tiene el alcance de cientos de miles de usuarios.

Los anuncios de Facebook, aunque no son gratuitos, ofrecen una gran oportunidad para llegar a los datos demográficos correctos para promocionar los servicios para tramites online que ofrece el SRI. Siempre que conozca bien a su cliente, puede usar métricas como intereses, ubicación geográfica, estado civil, edad y muchos otros, para ubicar a los posibles consumidores y enviarlos a páginas de destino estratégicamente dirigidas, también conocidas como páginas de restricción.

\section{Referencias bibliográficas}

Arévalo, J., \& Crespo, A. (Mayo de 2016). Modelo de devolución del IVA para disminuir la evasión fiscal. Obtenido de http://repositorio.ug.edu.ec/handle/redug/10958

Armijos, N. (2019). Marketing digital: una herramienta para potenciar y promocionar las Mipymes en el ámbito internacional.

Estrella, J. (22 de Octubre de 2014). Importancia de la gestión del servicio de Rentas Internas en los impuestos directos y la distribución de la riqueza, período 20072012.

Ferrell, O. \& Hartline, M. (2014). Estrategias de Marketing. México D.F, México: Cengage.

Gutiérrez, C. y Nava, R. (2016). Mercadotecnia digital y las medianas empresas: revisión de la literatura. Revista venezolana de innovación, tecnología y conocimiento. V. 13(1), pp. 45-61.

SRI. (Julio de 2016). Mi guía tributaria. Impuesto al Valor Agregado (IVA). Obtenido de http://multimedia.sri.gob.ec/flipbook/guia-2.html

SRInforma. (2016). Facturación. ¿Cómo emito comprobantes de venta? Obtenido de https://www.sri.gob.ec/DocumentosAlfrescoPortlet/descargar/c35f2e99-71144c24-aa0c-faa5d94d1baa/Triptico\%20Facturacion.pdf

SRInforma. (2016). REQUISITOS. Inscripción, actualización y cierre. Registro Único de Contribuyentes (RUC). Obtenido de https://www.sri.gob.ec/DocumentosAlfrescoPortlet/descargar/53089cd9-38c945ec-943a-b63e212f9373/NAC-DGERCGC1700000587\%20S.R.O.\%20\%20142\%20DE\%2018-12-2017.pdf 